Original

\title{
Alpha2u-Globulin Nephropathy in Rats Treated with a Glycosaminoglycan Extracted from Sea Cucumber
}

\author{
Kazuo Hakoi ${ }^{1,3}$, Taiji Hayashi ${ }^{1}$, Kenji Irimura ${ }^{1}$, Shuji Hayashi ${ }^{1,3}$, Satoshi Suzuki ${ }^{1}$, \\ Shuji Yamaguchi ${ }^{1}$, Noboru Konishi ${ }^{2}$, and Shoji Fukushima ${ }^{3}$ \\ ${ }^{1}$ Drug Safety Laboratory, Taiho Pharmaceutical Co., Ltd., 224-2 Hiraishi-ebisuno, Kawauchi-cho, Tokushima 771-0194, \\ Japan \\ ${ }^{2}$ Second Department of Pathology, Nara Medical University, 840 Shijo-cho Kashihara, Nara 634-8521, Japan \\ ${ }^{3}$ First Department of Pathology, Osaka City University Medical School, 1-4-3 Asahi-cho, Abeno-ku, Osaka 545-8585, \\ Japan
}

\begin{abstract}
DHG, a glycosaminoglycan extracted from the sea cucumber is known to show antithrombotic effects in animals and be a possible therapeutic agent for prevention of disseminated intravascular coagulation. In toxicology study, however, dose-dependent intracellular deposition of eosinophilic or basophilic droplets, and crystalloid substances were observed in the proximal tubular epithelial cells of the kidneys when male Sprague-Dawley rats were administered DHG daily for two weeks at the doses of 10,100 , and $200 \mathrm{mg} / \mathrm{kg}$. In contrast to heparin, a glycosaminoglycan that has been reported to induced similar deposits which were identified to be composed of a hemoglobin-like substance using the alizarin red staining. The inclusions observed in rats given DHG were immunohistochemically positive for $\alpha 2 u-$ globulin. Moreover, the droplets showed metachromasia in toluidine blue staining, and negative for Hall's, Gmelin's, Schmol's tests, and alizarin red staining, but the crystalloids were positive for alizarin red staining. These results suggested that DHG-induced degeneration of proximal tubular epithelial cells in male rats is related to $\alpha 2 \mathrm{u}$-globulin accumulation. (J Toxicol Pathol 2001; 14: 13-18)
\end{abstract}

Key words: $\alpha 2 \mathrm{u}$-globulin nephropathy, glycosaminoglycan, kidney, rat

\section{Introduction}

DHG (Cord number in Taiho Pharmaceutical Co., Ltd.), a depolymerized fragment of glycosaminoglycan extracted from the surface of the body of sea cucumber, Stichopus japonicus Selenka has antithrombotic effects and is now under development as a new alternate antithrombotic and anticoagulant agent of heparin at Taiho Pharmaceutical Co., Ltd.

A repeated intravenous toxicity study in rats performed as a series of safety evaluation of DHG revealed swelling of Kupffer's cells in the liver in both sexes and inclusion of droplets and needle-like crystalloid substances in the cytoplasm of proximal tubular epithelia in the kidneys of males (in-house document). It has been reported that these changes in the liver and kidneys were observed in the toxicity studies of this class of drugs ${ }^{1,2}$. Briefly, swelling of Kupffer's cells in the liver is a morphological change which

Received: 12 October 2000, Accepted: 1 December 2000 Mailng address: Kazuo Hakoi, Drug Safety Laboratory, Taiho Pharmaceutical Co., Ltd., 224-2 Hiraishi-ebisuno, Kawauchi-cho, Tokushima 771-0194, Japan

TEL: 81-88-665-5866 FAX: 81-88-665-5692

E-mail: kazuo-hakoi@taiho.co.jp reflected the biodegradation process of the test article, a glycosaminoglycan ${ }^{2}$. Crystalloid substances in the cytoplasm of proximal tubular epithelia in the kidneys in males have been considered to be reabsorbed hemoglobin produced by hemorrhage due to antithrombotic action of the test article, because the crystalloid substances were positive for alizarin red staining².

Droplets in the cytoplasm of proximal tubular epithelia in the kidneys were also observed at the lower doses of DHG where no hemorrhagic changes occurred, and this change was observed only in males. Therefore, $\alpha 2 u$-globulin, which has recently received attention because of the cause of nephropathy in male rats ${ }^{3,4}$, was suspected in the involvement of this change.

Thus, in order to examine the toxicological meaning of proximal tubular lesions in the kidneys of rats induced by administration of DHG, toxicologic pathological evaluation was performed in the liver, an anabolic organ, and the kidneys, an excretory organ of $\alpha 2 \mathrm{u}$-globulin.

\section{Materials and Methods}

Animals and housing conditions

Crj: CD (SD) SPF male rats at 5 weeks of age were 
purchased from Charles River Japan Inc. After quarantine and acclimation, 24 animals showing favorable growth and general conditions were used at 6 weeks of age in the present study. The animals were housed in an animal room environmentally maintained under the following conditions: temperature; $20-26^{\circ} \mathrm{C}$, humidity; 30-70\%, lighting: $12-\mathrm{hr}$ lighting (6 a.m.-6 p.m.)/dark cycle and frequency of ventilation; 10 times or more/hr. Two animals were housed in a stainless bracket-type cage (R-IS: $260 \mathrm{~mm} \times 380 \mathrm{~mm} \times$ $180 \mathrm{~mm}$, Japan CLEA, Inc.) throughout the study period. Each animal was identified with an ear tag inscribed with an identification number at arrival. The animals were given pellet diet (CE-2, Japan CLEA, Inc.) and tap water via an automated water supplier ad libitum.

Housing and handling of the animals were performed properly according to "Standards on rearing and housing of experimental animals (issued by the Prime Minister's Office in 1980)," "Guidelines for procedure of disposal of dead animals (Notification issued by the Prime Minister's Office in 1995)" and "Guidelines for animal experiments at Tokushima Research Center, Taiho Pharmaceutical Co., Ltd. (established in 1992).

\section{Experimental design}

DHG synthesized at Research Laboratory, Taiho Pharmaceutical Co., Ltd. was used in the present study. From a preliminary 2 -week study of DHG in rats at dose levels of 0,10 , and $100 \mathrm{mg} / \mathrm{kg}$, an increase of cytoplasmic droplets with degeneration in the proximal tubular epithelia of kidneys were observed at dose of $100 \mathrm{mg} / \mathrm{kg}$ (in-house document). Therefore, taking the duration of the administration period in the present nephrotoxicity study into account, $200 \mathrm{mg} / \mathrm{kg}$ was selected as the high dose level and 100,10 , and 0 (vehicle control group) $\mathrm{mg} / \mathrm{kg}$ were selected. Six animals were allocated to each group. The dosing solution was prepared by dissolving the test article in physiological saline to make $0.2,2$, and $4 \%$ solution. Intravenous administration was selected because of the expected clinical route of DHG. The dosing volume was 0.5 $\mathrm{mL} / 100 \mathrm{~g}$ body weight. The test article was administered once a day for 14 days at the dosing rate of $1 \mathrm{~mL} / \mathrm{min}$.

\section{General condition and body weight}

General conditions of all animals were observed twice a day, and body weight was measured three times a week.

\section{Autopsy and organ weight}

All animals were sacrificed by exsanguination under anesthesia with ether on day 14 . The liver and kidneys were weighed and the relative organ weights were calculated on the basis of body weight on the day of autopsy.

\section{Light and electron microscopy}

The liver and kidneys of all animals were fixed in $10 \%$ buffered formalin, and embedded in paraffin and stained with hematoxylin and eosin by using standard laboratory protocol. The specimens were examined under a light microscopy. A portion of the liver and kidneys was taken from 3 animals in each group. These samples were fixed in $2.5 \%$ glutaraldehyde, post-fixed in $1 \% \mathrm{OsO}_{4}$ solution, and embedded in Epon 812. The blocks were cut into ultra thin sections and double-stained with uranyl acetate and lead nitrate. These specimens were examined under an electron microscope, JEM1200EXII (JEOL).

\section{Histochemistry and immunohistochemistry}

As for substances included in the cytoplasm of Kupffer's cells in the liver and of proximal tubular epithelia in the kidneys identified by hematoxylin-eosin staining, histochemical and immunohistochemical examinations were performed using paraffin-specimens.

Histochemistry: Alcian blue-PAS ( $\mathrm{pH} 2.5$ ) reaction, toluidine blue staining were performed to identify glycosaminoglycan, and Berlin blue staining, alizarin red staining, Gmelin's test, Hall's test, and Schmorl's test were performed to identify endogenous pigments.

Immunohistochemisty: Immunohistochemical examination was performed by the method reported by Konishi et al. ${ }^{5}$ using anti- $\alpha 2 \mathrm{u}$-globulin antibody prepared at the Second Department of Pathology, Nara Medical University.

\section{Statistical analysis}

For body weight and organ weight, Bartlett's analysis for homogeneity of variance was performed followed by one-way analysis of variance in case of homogeneous variance or the Kruskal-Wallis test in case of heterogeneous variance. In case of significant results, the Dunnett's test for multiple group comparisons was performed.

\section{Results}

\section{General condition and body weight}

No significant changes were observed.

\section{Organ weight}

The liver and kidney weights are shown in Table 1. An increase in the relative weight of the kidneys was observed in the groups given 100 and $200 \mathrm{mg} / \mathrm{kg}$.

\section{Light and electron microscopy}

Histopathological findings of the liver and kidneys are shown in Table 2. Swelling of Kupffer's cells in the liver was observed in the groups given 100 and $200 \mathrm{mg} / \mathrm{kg}$. Foamy substances were observed in the swollen Kupffer's cells. Electron microscopical examination revealed that the foamy substances were in lysosomes.

Eosinophilic or basophilic droplets and vacuoles were observed in the cytoplasm of proximal tubular epithelia in the kidneys (Fig. 1) in a dose-dependent manner. Furthermore, needle-like crystalloid substances were observed in the cytoplasm of proximal tubular epithelia in the kidneys in the group given $200 \mathrm{mg} / \mathrm{kg}$. Electron microscopical examination revealed that droplets in the cytoplasm of proximal tubular 
Table 1. Final Body Weight and Organ Weight in Male Rats Injected with DHG

\begin{tabular}{|c|c|c|c|c|c|}
\hline \multirow[t]{3}{*}{ Organ } & \multirow{3}{*}{$\begin{array}{l}\text { Dose }(\mathrm{mg} / \mathrm{kg}) \\
\text { No. of animals }\end{array}$} & \multirow{2}{*}{$\begin{array}{c}\text { Saline } \\
0\end{array}$} & \multicolumn{3}{|c|}{ DHG } \\
\hline & & & 10 & 100 & 200 \\
\hline & & 6 & 6 & 6 & 6 \\
\hline Body weight & (g) & $234 \pm 14.1$ & $236 \pm 6.8$ & $218 \pm 10.4$ & $228 \pm 22.6$ \\
\hline \multirow[t]{2}{*}{ Liver } & $(\mathrm{g})$ & $7.40 \pm 0.81$ & $7.47 \pm 0.55$ & $7.40 \pm 0.60$ & $7.48 \pm 1.02$ \\
\hline & $(\mathrm{g} / 100 \mathrm{gBW})$ & $3.16 \pm 0.17$ & $3.17 \pm 0.28$ & $3.40 \pm 0.18$ & $3.27 \pm 0.15$ \\
\hline \multirow[t]{2}{*}{ Kidney-R } & (g) & $1.01 \pm 0.14$ & $1.02 \pm 0.05$ & $1.03 \pm 0.07$ & $1.12 \pm 0.12$ \\
\hline & $(\mathrm{g} / 100 \mathrm{gBW})$ & $0.43 \pm 0.05$ & $0.43 \pm 0.02$ & $0.48 \pm 0.03^{*}$ & $0.49 \pm 0.03^{* *}$ \\
\hline \multirow[t]{2}{*}{ Kidney-L } & (g) & $1.04 \pm 0.11$ & $1.05 \pm 0.06$ & $1.04 \pm 0.07$ & $1.13 \pm 0.12$ \\
\hline & $(\mathrm{g} / 100 \mathrm{gBW})$ & $0.44 \pm 0.02$ & $0.44 \pm 0.02$ & $0.48 \pm 0.02 *$ & $0.50 \pm 0.03 * *$ \\
\hline
\end{tabular}

Results are given as the mean \pm S.D.

Significantly different from the saline control : $* \mathrm{P}<0.05$, $* * \mathrm{P}<0.01$

Table 2. Histopathological Findings in Male Rat Liver and Kidney Injected with DHG

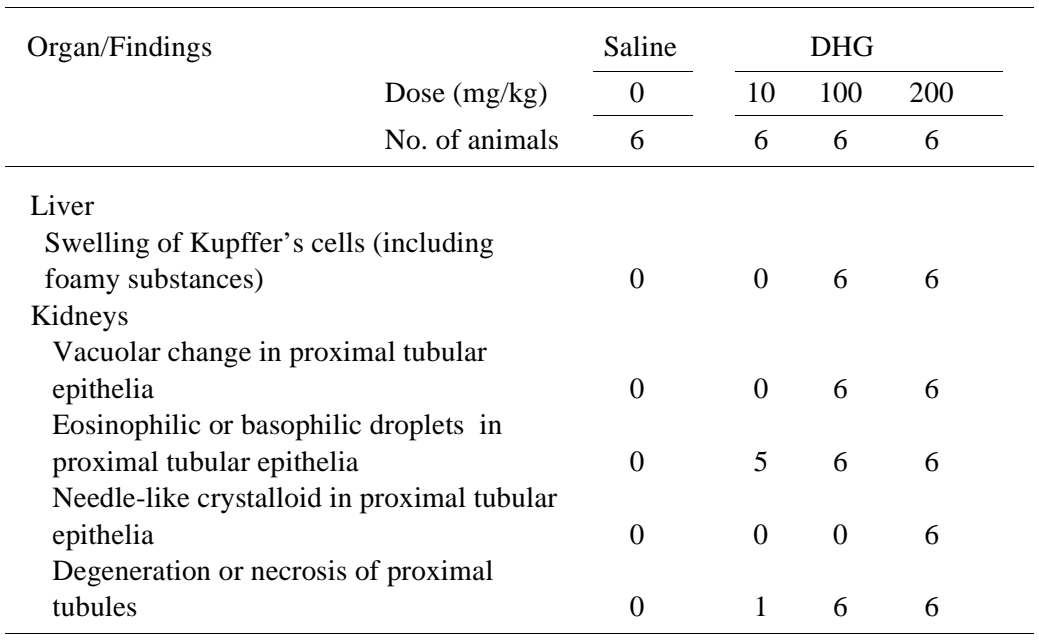

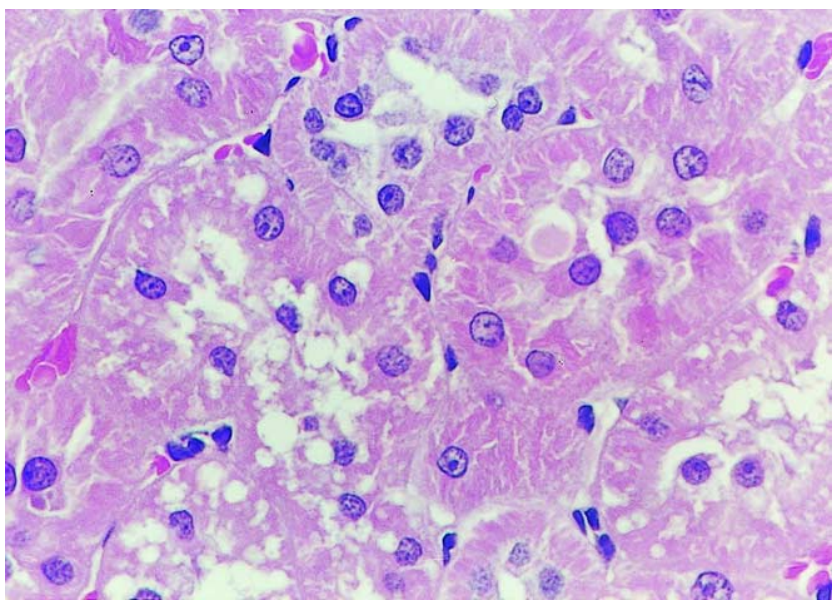

Fig. 1. Degeneration of proximal tubules with eosinophilic droplets or vacuoles observed in the kidney injected with DHG 200 $\mathrm{mg} / \mathrm{kg}, \mathrm{HE} \times 400$.

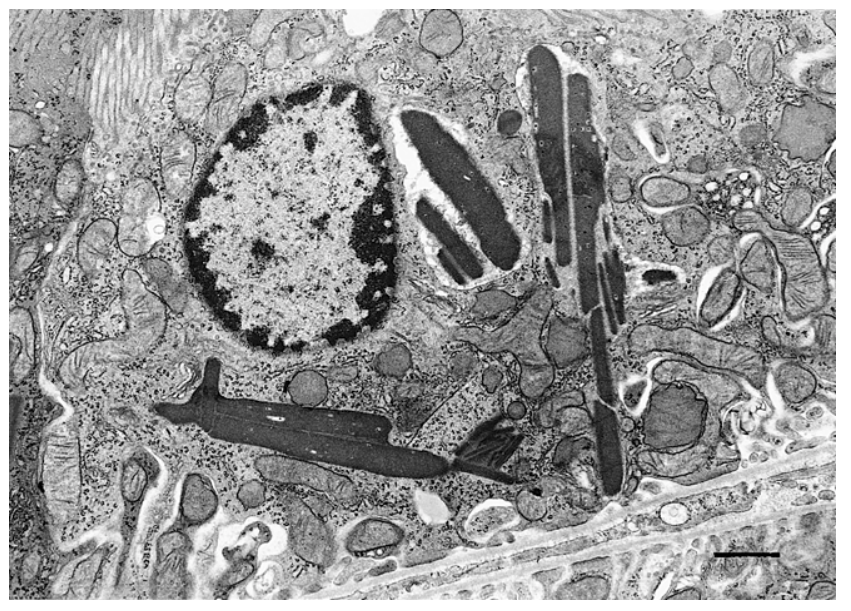

Fig. 2. Electron-micrograph of a proximal tubular cell of DHGtreated male rat. Crystalloid and electron-dense substances were observed in lysosomes injected with DHG $200 \mathrm{mg} / \mathrm{kg}, \times$ 6,000, Bar $=1 \mu \mathrm{m}$. 
Table 3. Histochemical and Immunohistochemical Staining for Inclusions in Male Rat Liver and Kidney Injected with DHG

\begin{tabular}{|c|c|c|c|c|}
\hline \multirow{3}{*}{$\begin{array}{l}\text { Organ } \\
\text { Site } \\
\text { Shape of inclusions }\end{array}$} & \multirow{3}{*}{$\begin{array}{c}\text { Liver } \\
\text { Kupffer's cell } \\
\text { Foamy }\end{array}$} & \multicolumn{3}{|c|}{ Kidney } \\
\hline & & \multicolumn{3}{|c|}{ Proximal tubular epithelia } \\
\hline & & $\begin{array}{l}\text { Vacuoles } \\
\text { (Granular) }\end{array}$ & Droplets & $\begin{array}{c}\text { Crystalloid } \\
\text { (Needle) }\end{array}$ \\
\hline \multicolumn{5}{|l|}{ Histochemistry } \\
\hline Alizarin red & - & - & - & + \\
\hline Alcian blue-PAS & + & + & + & - \\
\hline Toluidine blue & $+\left(\mathrm{M}^{+}\right)$ & + & $+\left(\mathrm{M}^{+}\right)$ & $+\left(\mathrm{M}^{+}\right.$or $\left.\mathrm{M}^{-}\right)$ \\
\hline Berlin blue & - & - & - & - \\
\hline Gmelin's test & - & - & - & - \\
\hline Hall's test & - & - & - & - \\
\hline Schmorl's test & - & - & - & - \\
\hline \multicolumn{5}{|c|}{ Immunohistochemistry } \\
\hline$\alpha 2 u$-globulin & - & - & + & + \\
\hline
\end{tabular}

+: positive, -: negative, $\mathrm{M}^{+}$: positive for metachromasia, $\mathrm{M}^{-}$: negative for metachromasia

epithelia were phagolysosomes and that needle-like crystalloid substances existed in lysosomes (Fig. 2).

\section{Histochemistry and immunohistochemistry}

Histochemical and immunohistochemical staining for the inclusions in the cytoplasm of Kupffer's cells in the liver and of proximal tubular epithelia in the kidneys are shown in Table 3.

Histochemistry: Foamy substances in Kupffer's cells in the liver and substances in vacuoles in proximal tubular epithelia in the kidneys were red purple to blue purple in color in Alcian blue-PAS staining and positive toluidine blue staining or metachromasia.

Eosinophilic or basophilic droplets in proximal tubular epithelia in the kidneys showed metachromasia in toluidine blue staining and negative alizarin red staining. In crystalloid substances showing positive alizarin red staining, toluidine blue staining, some showed metachromasia and some did not. Furthermore, droplets in the cytoplasm of proximal tubular epithelia in the kidneys were red purple or blue purple in color in Alcian blue-PAS staining.

Besides, Berlin blue reaction, Gmelin's test, Hall's test, and Schmorl's test were negative in the liver and kidneys of the animals treated with DHG.

Immunohistochemistry: In the immunohistochemical staining with rat anti- $\alpha 2 \mathrm{u}-$ globulin antibody, positive reaction was observed in the centrilobular area in the liver of the control and DHG treated groups. There was no difference in the intensity of staining among the groups. Foamy substances in Kupffer's cells in the DHG treated groups were negative in the immunohistochemical staining.

Immunohistochemically, microdroplets, probably lysosomes, in proximal tubular epithelia in the kidneys showed positive reaction in the control group. On the other hand, in the DHG-treated groups, needle-like crystalloid substances, and eosinophilic or basophilic droplets in the cytoplasm of tubular epithelia in the kidneys showed

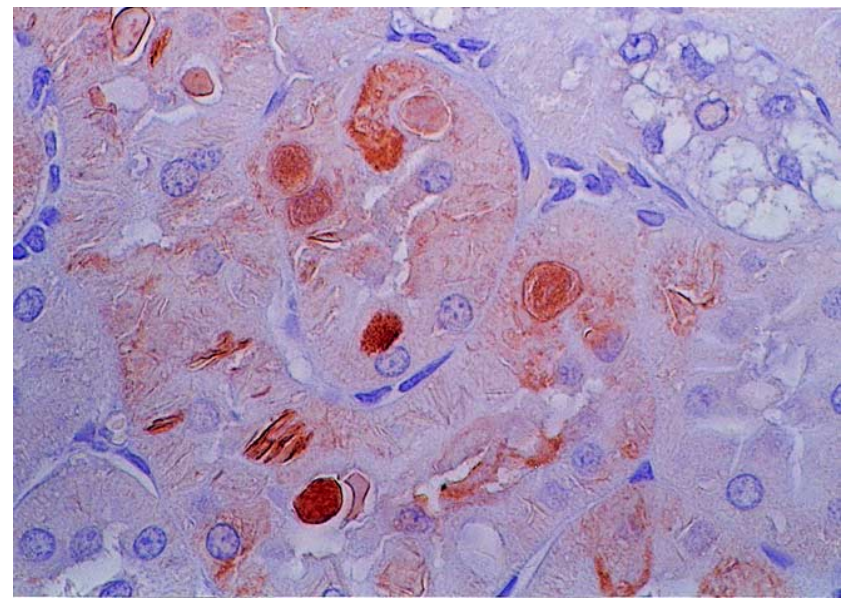

Fig. 3. Immunohistochemical staining of the kidney injected with DHG $200 \mathrm{mg} / \mathrm{kg}$. The $\alpha 2 \mathrm{u}$-globulin positive droplets and crystalloid substances were observed in the cytoplasm of proximal tubular cells, but not in the vacuolated tubules, $\times$ 400 .

positive reaction, but vacuoles in the cytoplasm showed negative reaction (Fig. 3).

\section{Discussion}

In rats, $\alpha 2 \mathrm{u}$-globulin is synthesized in the liver ${ }^{6}$. In the immunohistochemical staining with anti- $\alpha 2 \mathrm{u}$-globulin in the liver, positive reaction was observed in the centrilobular area both in the control and DHG treated groups, and there was no difference in the intensity of staining among the groups.

On the other hand, in the DHG treated groups, Kupffer's cells were swollen and foamy substances were observed in their cytoplasm. Foamy substances were considered to include an acidic glycosaminoglycan, because these substances were red purple to blue purple in color in Alcian blue-PAS staining and metachromasia in toluidine 
blue staining. Reticuloendothelial tissues such as Kupffer's cells play a major role in the disposal of acidic glycosaminoglycans such as heparin ${ }^{7}$. Therefore, the inclusions in Kupffer's cells observed in administration of DHG, an acidic glycosaminoglycan, were considered to be substances derived from DHG, and this histopathological finding was considered to be a change reflecting the disposal of DHG from the body.

In the toxicity studies of heparin, low molecular weight heparin in rats, it has been reported that crystalloid substances were observed in the cytoplasm of proximal tubular epithelia in the kidneys of male rats ${ }^{2}$. Since the crystalloid substances were positive for alizarin red staining, the alizarin has a high affinity to hemoglobin, these substances are considered to be reabsorbed hemoglobin produced by hemorrhage, a side-effect of drugs ${ }^{2}$. In the DHG treated groups, eosinophilic or basophilic droplets in the cytoplasm of proximal tubular epithelia were observed from low dose where no hemorrhagic changes occurred. Furthermore, the droplets were red purple to blue purple in color in Alcian blue-PAS staining, and some of the inclusions showed metachromasia in toluidine blue staining. Therefore, it was considered that the characteristics of these droplets were different from those of eosinophilic body and hyaline droplets observed in normal animals ${ }^{8}$. DHG is excreted slowly from the kidneys in the same way as the related drugs ${ }^{9}$ and the concentration of DHG in the kidneys is higher than in other organs in the pharmacokinetics study of DHG (in-house document). Therefore, the possibility that these mucus-staining positive droplets and crystalloid substances in the cytoplasm of tubular epithelia include substances derived from reabsorbed DHG (modified DHG substances) is most likely.

Crystalloid substances in the cytoplasm produced by DHG administration were observed in lysosomes around the $\mathrm{P} 2$ area of proximal tubules in the kidneys. The P2 area in proximal tubules in the kidneys plays an important role in the reabsorption and metabolism of low molecular proteins ${ }^{10}$, and is known as the reabsorption and storage place for a protein peculiar to male rats ( $\alpha 2 \mathrm{u}$-globulin), which is able to bind easily to various kinds of chemicals and proteins ${ }^{11}$. It has been reported that the proximal tubules of the kidneys of male rats are injured often by the inclusion of this protein to result in $\alpha 2 \mathrm{u}$-globulin nephropathy ${ }^{12,13}$. Thus, the immunohistochemical staining for $\alpha 2 \mathrm{u}$-globulin was performed, and eosinophilic or basophilic droplets and needle-like crystalloid substances showed positive reaction, indicating the presence of $\alpha 2 \mathrm{u}$-globulin. Degenerative necrosis in proximal tubules of the kidneys observed in administration of DHG coincided mostly with the presence of these inclusions. Therefore, the renal lesions in male rats induced by DHG treatment were presumed to be caused by the inclusion of large molecules, which were formed by binding DHG-derived substances to $\alpha 2 \mathrm{u}$-globulin during the excretory process of DHG to urine, in lysosomes of renal tubules (excretory disturbance ${ }^{14}$ ).

$\alpha 2 \mathrm{u}$-globulin is not synthesized in guinea pigs, monkeys, and dogs, and the presence of $\alpha 2 \mathrm{u}$-globulin has not been proved in mice and humans ${ }^{3,4,12}$. $\alpha 2 \mathrm{u}$-globulin is synthesized mainly in the liver of male rats ${ }^{6,12}$, and in female rats, $\alpha 2 \mathrm{u}$-globulin is not synthesized ${ }^{15}$, or if synthesized, the amount of $\alpha 2 \mathrm{u}$-globulin synthesized is $1 / 120$ that in male rats $^{16}$. Therefore, it has been reported that there are species and gender differences in nephropathy caused by the inclusion of $\alpha 2 \mathrm{u}-\mathrm{globulin}$ in the proximal tubular epithelia $^{12,17}$. With the administration of DHG, the inclusions in the cytoplasm of proximal tubular epithelia were also observed in male rats, but not in female rats and dogs (in-house document). Therefore, these renal lesions in male rats could be evaluated toxicologically to be changes related to the renal function peculiar to male rats.

Although it has been reported that $\alpha 2 \mathrm{u}$-globulin nephropathy is induced by administration of various chemicals $^{18-35}$, we are confident that this is the first report that $\alpha 2 \mathrm{u}-\mathrm{globulin}$ nephropathy was caused by a glycosaminoglycan.

\section{References}

1. Hirakawa $T$, Nagashima $Y$, Tamura $K$, Nishimura $N$, Sugimoto K, Utunomiya T, and Kamata T. Subacute toxicity study of heparin like substance in rats (in Japanese). The Clinical Report ${ }^{\circledR}$ 1984; 18: 5249-5265.

2. Shibata N, Kiyosawa G, Isaka T, Momose Y, Isaji M, Ohba M, Ozawa S, Naito J, and Kuwahara N. Toxicity study of low molecular weight heparin sodium (FR-860) (2nd report)-Subacute intravenous toxicity study in rats followed by a recovery study (in Japanese). The Clinical Report ${ }^{\circledR}$ 1989; 23: 6129-6207.

3. Alden CL. A review of unique male rat hydrocarbon nephropathy. Toxicol Pathol 1986; 14: 109-111.

4. Hard GC. Mechanisms of chemically induced renal carcinogenesis in the laboratory rodent. Toxicol Pathol 1998; 26: 104-112.

5. Konishi N, Nishii K, Hayashi I, Nakaoka S, Matsumoto K, Yabuno T, Kitahori Y, and Hiasa Y. Inhibitory effect of citrate on rat renal tumors induced by N-ethyl-Nhydroxyethylnitrosamine followed by potassium dibasic phosphate. Jpn J Cancer Res 1993; 84: 128-134.

6. Mancini MA, Chatterjee B, and Roy AK. Age-depandent reversal of the lobular distribution of androgen-inducible $\alpha 2 \mathrm{u}$-globulin and androgen-repressible SMP-2 in rat liver. J Histochem and Cytpchem 1991; 39: 401-405.

7. Watanabe J, Hori K, Iwamoto K, and Ozeki S. Disposition of tritium-labelled heparin in rats. J Pharm Dyn 1982; 5: 627-637.

8. Uwagawa S, Saito K, Nakayama A, Umihira M, and Okuno Y. Comparison of hyaline droplets in rats with chronic progressive nephropathy and chemical-induced $\alpha 2 u-$ globulin nephropathy. J Toxicol Pathol 1992; 5: 195-203.

9. Nishiyama M, Matubara Y, and Ikeda S. Studies on the matabolic fate of low molecular weight heparin (FR-860) (3rd report)-Repeated administration for 21 days (in Japanese). The Clinical Report ${ }^{\circledR}$ 1989; 23: 6495-6501.

10. Straus W. Cytochemical observation on the relationship of lysosomes and phagosomes in kidney and liver by staining for acid phosphatase and intravenously injected horseradish 
peroxidase. J Cell Biol 1964; 20: 27-49.

11. Short BG, Burnett VL, Cox MG, Bus JS, and Swenberg JA. Site-specific renal cytotoxicity and cell proliferation in male rats exposed to petroleum hydrocarbons. Lab Invest 1987; 57: 564-577.

12. Borghoff SJ, Short BG, and Swenberg JA. Biochemical mechanisms and pathobiology of $\alpha 2 \mathrm{u}$-globulin nephropathy. Annu Rev Pharmacol Toxicol 1990; 30: 49-67.

13. Short BG, Burnett VL, and Swenberg JA. Histopathology and cell proliferation induced by 2,2,4-trimethylpentane in the male rat kidney. Toxicol Pathol 1986; 14: 194-203.

14. Lehman-McKeeman LD, Rivera-Torres MI, and Caudill D. Lysosomal degeneration of $\alpha 2 \mathrm{u}$-globulin and $\alpha 2 \mathrm{u}$-globulinxenobiotic conjugates. Toxicol Appl Pharmacol 1990; 103: 539-548.

15. Burnet VL. Localization of $\alpha 2 \mathrm{u}-$ globulin within protein droplets of male rat kidney, Immunohistochemistry using perfusion-fixed, GMA-embedded sections. J Histochem Cytochem 1989; 37: 813-818.

16. Saito K, Uwagawa S, Kaneko H, and Yoshitake A. Behavior of $\alpha 2 \mathrm{u}$-globulin accumulating in kidneys of male rats treated with $d$-limonene: Kidney-type $\alpha 2 \mathrm{u}$-globulin in the urine as a marker of $d$-limonene nephropathy. Toxicology 1991; 79: 173-183.

17. Vandoren G. Different forms of alpha $\alpha 2 \mathrm{u}$ globulin in male and female rat urine. Eur J Biochem 1983; 134: 175-181.

18. Blumbach K, Pahler A, Deger HM, and Dekant W. Biotrasformation and male rat-specific renal toxicity of diethyl ethyl- and dimethyl methylphosphonate. Toxicol Sci 2000; 53: 24-32.

19. Borghoff SJ, Youtsey NL, and Swenberg JA. A comparison of European high test gasoline and PS-6 unleaded gasoline in their abilities to induce $\alpha 2 \mathrm{u}$-globulin nephropathy and renal cell proliferation. Toxicol Lett 1992; 63: 21-33.

20. Caldwell DJ, Eldridge SR, Lington AW, and McKee RH. Retrospective evaluation of $\alpha 2 \mathrm{u}$-globulin accumulation in male rat kidneys following high doses of diisononyl phthalate. Toxicol Sci 1999; 51: 153-160.

21. Diertrich DR, and Swenberg JA. The presence of $\alpha 2 \mathrm{u}-$ globulin is necessary for $d$-limonene promotion of male rat kidney tumors. Cancer Res 1991; 51: 3512-3521.

22. Dominick MA, Robertson DG, Bleavins MR, Sigler RE, Bobrowski WF, and Gough AW. $\alpha 2 \mathrm{u}$-globulin nephropathy without nephrocarcinogenesis in male Wistar rats administered 1-(aminomethyl)cyclohexaneacetic acid. Toxicol Appl Pharmacol 1991; 111: 375-387.

23. Hard GC, and Whysner J. Risk assessment of $d$-limonene: an example of male rat-specific renal tumorigens. Crit Rev
Toxicol 1994; 24: 231-254.

24. Kim S, Qualls CW Jr, Reddy G, and Stair EL. 1,3,5Trinitrobenzene-induced $\alpha 2 \mathrm{u}$-globulin nephropathy. Toxicol Pathol 1997; 25: 195-201.

25. Lehman-McKeeman LD, Rodriguez PA, Caudill D, Fey ML, Eddy CL, and Asquith TN. Hyaline droplet nephropathy resulting from exposure to 3,5,5-trimethylhexanoyloxybenzene. Toxicol Appl Pharmacol 1991; 107: 429-438.

26. Medinsky MA, Wolf DC, Cattley RC, Wong B, Janszen DB, Farris GM, Wright GA, and Bond JA. Effects of thirteenweek inhalation exposure to ethyl tertiary butyl ether on fischer-F344 rats and CD-1 mice. Toxicol Sci 1999; 51: $108-118$.

27. Murty CV, Olson MJ, Garg BD, and Roy AK. Hydrocarboninduced hyaline droplet nephropathy in male rats during senescence. Toxicol Appl Pharmacol 1988; 96: 380-392.

28. Pillip RD, and Egan GF. Effect of C10-C11 isoparaffinic solvent on kidney function in Fischer 344 rats during eight weeks of inhalation. Toxicol Appl Pharmacol 1984; 73: 500-510.

29. Prescott-Mathews JS, Poet TS, and Borghoff SJ. Evaluation of the in vivo interaction of methyl tert-butyl ether with $\alpha 2 u-$ globulin in male F-344 rats. Toxicol Appl Pharmacol 1999; 157: 60-67.

30. Rasonyi T, Schlatter J, and Dietrich DR. The role of $\alpha 2 u-$ globulin in ochratoxin A induced renal toxicity and tumoes in F344 rats. Toxicol Lett 1999; 104: 83-92.

31. Short BG, Burnett VL, and Swenberg JA. Elevated proliferation of proximal tubule cells and localization of accumulated $\alpha 2 \mathrm{u}$-globulin in F344 rats during chronic exposure to unleaded gasoline or 2,2,4-trimethylpentane. Toxicol Appl Pharmacol 1989; 101: 414-431.

32. Stone LC, Kanerva RL, Burns JL, and Alden CL. Decalin induced nephrotoxicity: Light and electron microscopic examination of the effects of oral dosing on the development of kidney lesions in the rat. Food Chem Toxicol 1987; 25: $43-52$.

33. Swenberg JA, Short B, Borghoff S, Strasser J, and Charbonneau M. The comparative pathobiology of $\alpha 2 \mathrm{u}-$ globulin nephropathy. Toxicol Appl Pharmacol 1989; 97: 35-46.

34. Uwagawa S, Nakayama A, Okuno Y, and Kawasaki H. Exfoliated cells in the urine reflect transient and sustained elevation of cell proliferation in rat $\alpha 2 \mathrm{u}$-globulin nephropathy. J Toxicol Pathol 1999; 12: 59-64.

35. Viau C, Bernard A, Gueret F, Maldague P, Gengoux P, and Lauwerys R. Isoparaffinic solvent-induced nephrotoxicity in the rat. Toxicology 1986; 38: 227-240. 